in vivo $35: 1939-1943$ (2021)

doi:10.21873/invivo.12460

Review

\title{
Headache Reporting in Oncology Trials Depends on the Demographics of the Study Population
}

\author{
BIRTE J. WOLFF ${ }^{1}$, MATTHAEUS J. WOLFF' ${ }^{2}$ and JOHANNES E. WOLFF ${ }^{3}$ \\ ${ }^{1}$ Swedish Covenant Hospital Department of Gynecology, Chicago, IL, U.S.A.; \\ ${ }^{2}$ Bellevue, WA, U.S.A.; \\ ${ }^{3}$ Wauconda, IL, U.S.A.
}

\begin{abstract}
Background/Aim: The frequency of adverse events (AEs) in clinical trials without control arms is difficult to interpret. Materials and Methods: This is a systematic literature review of AEs reported from the placebo arms of randomized cancer trials in PubMed between 2008 and 2020. Results: We found 80 placebo patient cohorts in 73 publications, describing 17,968 subjects who received placebo. Headaches were reported in 35 patient cohorts with an average frequency of $12.3 \%(+/-S D=8.0$, range $=0.4$ $34.1)$, and were more common in cohorts with a median age between 45 and 50 years, with higher performance status, and breast cancer (average 29.8\% +/- SD=6.1). AEs leading to discontinuation were reported in $5 \%$ of cohorts $(+/-S D=5.1$, range=0-22.7). Conclusion: Considering covariates allows more accurate interpretation of the observed AE frequencies in cancer trials.
\end{abstract}

The overall benefit of oncology medicines can be seen as the net after side effects have been subtracted from efficacy. During oncology drug development, the therapeutic index in preclinical experiments (1) is translated into a formal benefit risk evaluation in clinical trials (2). Both compare the findings of the drug with those without the drug. However, there is a gap in the data production during early drug development, when clinical trials have no placebo control arms (3). It is reasonable to assume a benefit of the drug, when a tumor shrinks in response to a novel agent. However, it is much harder to judge

This article is freely accessible online.

Correspondence to: Dr. Med habil Johannes E. Wolff, MD, Ph.D., 1709 Napa Suwe Ln, Wauconda, IL 60084, U.S.A. E-mail: johannesewolff@gmail.com

Key Words: Meta-analysis, systematic review, adverse events, placebo, cancer, review. the observed frequency of an adverse event (AE) without a control group. The observation could be compared to the expected frequency in the patient population without the agent under investigation - if only that baseline was known.

AEs observed in placebo arms of randomized trials are potential sources and $\mathrm{AE}$ literature reviews have recently contributed to drug development (4). Interestingly, there was a high level of heterogeneity, and a correlation between $\mathrm{AE}$ frequencies in placebo groups and those in treatment arms (5). This could reflect the effect of the diagnosis and patient demographics (6).

A previous similar analysis of AEs in placebo arms (5) identified 10 trials published between 2006 and 2018; the most frequent grade 3 to 4 AEs in placebo patients was hypertension $(2.8 \%)$. We aimed to build upon these findings, analyzing specific AEs in more granularity, and hypothesized that demographic variables of the patient population influence the reporting even among placebo arms. To address this hypothesis, we expanded the data collection, and selected one specific AE: headache. Headache is among the most commonly reported AEs, there are only three grades, and synonyms are rarely used.

\section{Materials and Methods}

The selection of included publications built upon a previous metaanalysis 2000-2018 (5), and used the 10 publications listed there. In addition, the search was updated for Jan 2018 - Nov 2020 in PubMed using the same method with one notable expansion: patient cohorts with measurable tumors were not excluded. We found a total of 1,597 titles, which eventually resulted in the inclusion of 73 publications. The search algorithm and list of included articles is available from the corresponding author. SPSS (Statistical Package for Social Studies, IBM version 23.0) and Excel (7) were used to conduct the analyses.

All analyses were exploratory in nature, and $p$-values were not considered confirmatory. We used ANOVA to compare the frequency of AEs among subgroups defined by categorical variables, such as cancer indication. For quantitative variables, we used linear regression and visual evaluation in scatter plots; and we 
transferred the quantitative variables to categorical variables to use ANOVA. This was first done for data from the placebo arms only, and then repeated as sensitivity analysis for all data, including the treatment arms of the studies.

\section{Results}

The selection resulted in 73 publications describing 161 cohorts (study arms) with a total sum of 41,836 documented persons. Among those were 35 placebo arms, in which headaches were reported. The grade of headache was reported in different ways: Most commonly the authors combined all grades as a single number (grade 1 and higher: 22 of 80 placebo arms), others reported individual grades, which allowed calculating the same value in further 12 , resulting in 34 study cohorts with a total number of 8,212 subjects for the main part of this analysis.

Interestingly, we found some smaller studies with accurate AE listing (8), whereas larger studies often applied a cut off of either a $5 \%$ or $10 \%$ frequency. Rare terms "feeling jittery" appear more often in studies with small numbers, that report every single patient complaint (8). In blinded studies, the investigators are not aware if the patient received the active drug, nonetheless AEs apparently caused by the drug are also reported in the placebo arms (9). The following analysis was restricted to $\mathrm{AE}$ frequencies regardless of causality.

Among the placebo monotherapy treated cohorts, the tumor status at treatment start was: no measurable tumor: $31.3 \%$, measurable tumor: $28.7 \%$, and mixed tumor status: $40 \%$. Cohorts with newly diagnosed malignancies reflected $37.5 \%$ of the database, relapsed or refractory $(\mathrm{R} / \mathrm{R})$ disease $47.5 \%$, and in $10 \%$ both were allowed. Healthy volunteers, and cancer prevention studies represented $2.5 \%$ each. Most of the cohorts documented no prior therapy lines $(n=28)$, while 17 studies had a median of one prior treatment line, four had a median of 2, three cohorts had a median of 3 and one trial had a median of 4 prior treatment lines.

The diagnoses and cancer locations were colorectal, liver, and lung: 9 each; hematologic and ovaries: 7 each; kidney, prostate, skin, and soft tissue: 5 each; breast: 4; brain, bone, gastric, gastrointestinal stromal tumor (GIST) pancreas, and no cancer (prevention): 2 each; bladder, head and neck, and esophagus: 1 each. The gender distribution was reported in 73 cohorts with an average of $55.2 \%$ male (range 0 in ovarian cancer to 100 in prostate cancer). The median age was documented in 76 cohorts, and the average was 57.7 years (range $=7.1-74, \mathrm{SD}=12.4$ years). The performance status at study entry was described in 65 cohorts by Eastern Cooperative Oncology Group (ECOG) status (10). ECOG status of 0 was reported between $5.2 \%$ and $100 \%$ with an average of $57.3 \%(\mathrm{SD}=24.6 \%)$. Placebo was provided by mouth (PO) in $73.8 \%$, intravenous (IV) in $17.5 \%$, subcutaneous (SC) in $5 \%$, intramuscular (IM) in $2.5 \%$, and

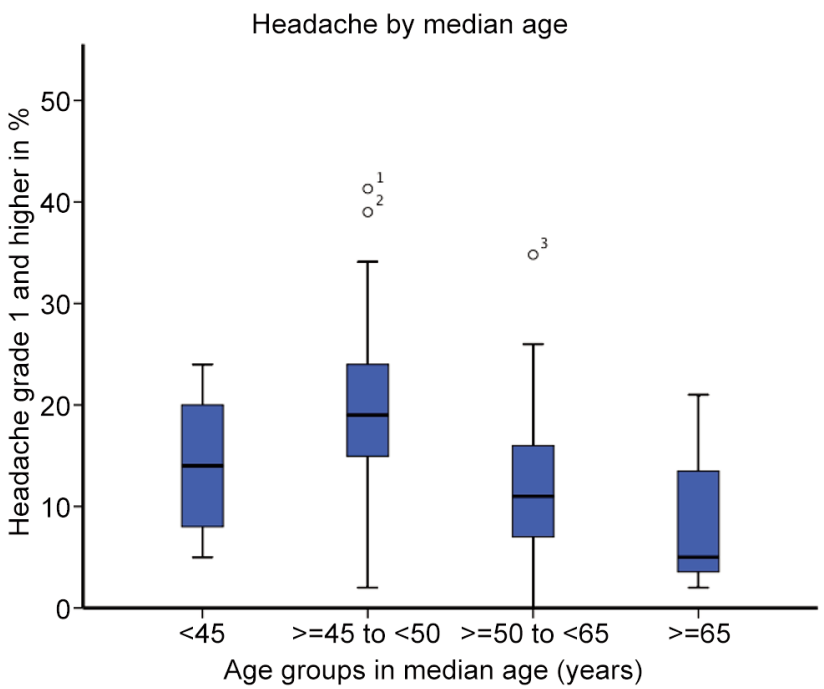

Figure 1. Influence of age on headache reporting in placebo arms only.

intradermal (ID) in $1.3 \%$. The studies were classified as phase I, phase II, or phase III in $2.5,27.5$, and $70 \%$, respectively.

Among the 80 placebo cohorts, the most commonly reported adverse event was fatigue [56], followed by nausea [57], vomiting [45], diarrhea [45], decreased appetite [37], headache [35], anemia [34], constipation [33], hypertension [32], and cough [32]. Among the 81 non-placebo cohorts, headache was reported with similar frequency [35 cohorts] but the other AEs differed: There was a trend for a higher frequency of diarrhea [63 cohorts], vomiting (49], constipation [40], thrombocytopenia [33], and neutropenia [26].

The average frequency of headache reported in the placebo arms was $12.3 \%(+/-\mathrm{SD}=8.0$, range $=0.4-34.1)$. When this value was weighted by patient numbers, the average was similar: $11.6 \%$. The estimator of effect size and heterogeneity using meta-analysis methods indicated that most of the variability was caused by real heterogeneity rather than chance: $\mathrm{I}^{2}=97.4 \%$, random model effect size $0.0809(8 \%)$. The frequency of treatment discontinuation caused by AEs was reported in 54 of the placebo arms with an average of 5.03\%, (range $=0-22.7, \mathrm{SD}=5.1 \%$ ). Severe adverse event (SAE) frequencies were reported in 37 cohorts: average $18.3 \%$ (range $=0-42, \mathrm{SD}=12.1 \%$ ). Among cohorts with investigational drugs, AE related discontinuation was $15 \%$ $(\mathrm{SD}=14.4)$, and SAEs $23.7 \%(\mathrm{SD}=13.8)$.

None of the included publications reported headache as an adverse drug reaction to the investigational agent, and within each study, the rate did not differ significantly between treatment and control arms. However, the average of the headache rates was moderately higher in the treatment groups (average \% 17.4 vs. 12.6). In comparison, the frequency of treatment discontinuation for AEs differed 

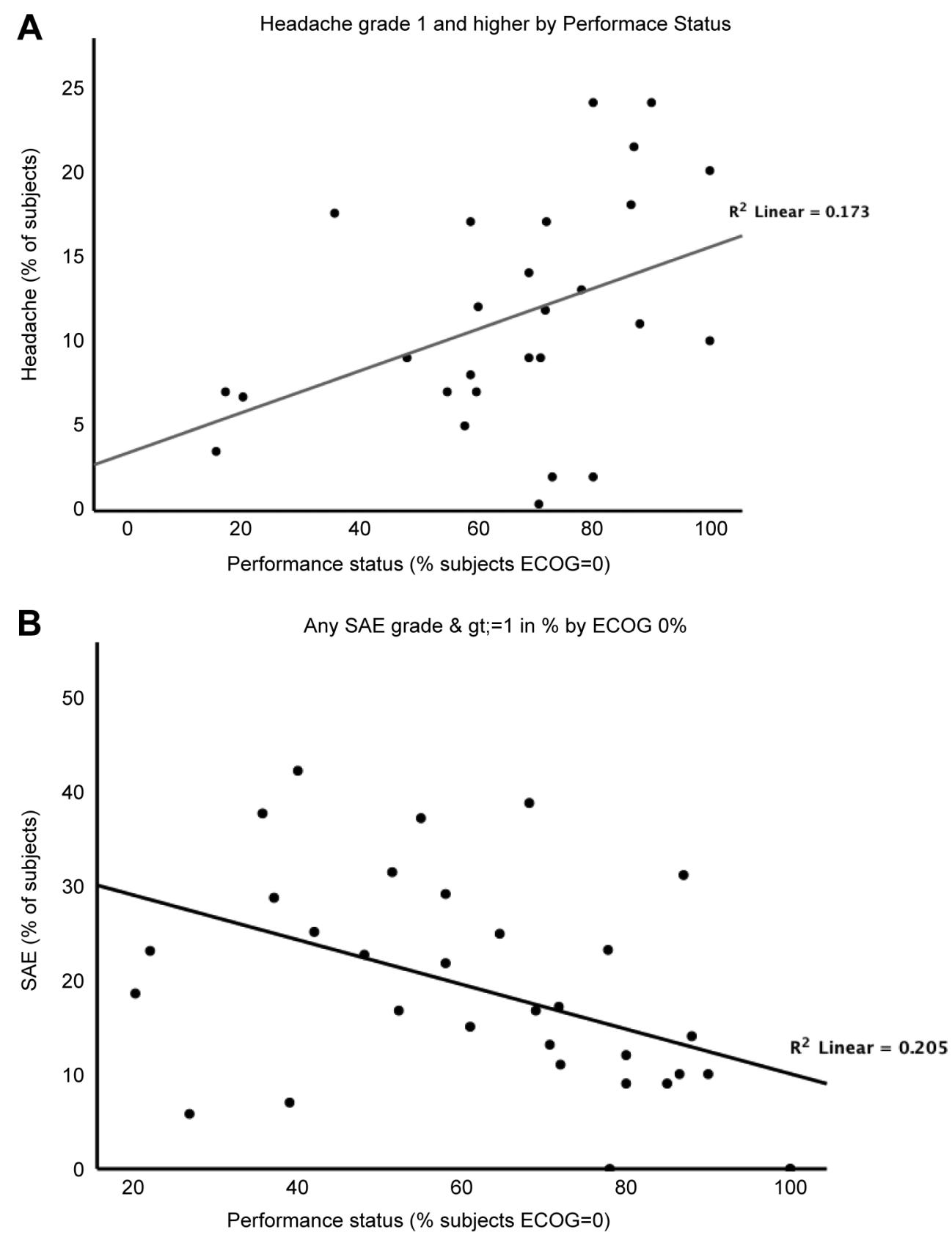

Figure 2. Eastern Cooperative Oncology Group (ECOG) status presented as \% patients with ECOG 0 versus adverse events among patient cohorts with placebo monotherapy. A) Headache, B) Severe adverse events (SAE).

largely between treatment and placebo arms $(14.4 \%$ in treatment arms vs. 5.1 in placebo, exploratory $p=0.000004)$.

Evaluating the influence of demographics on headache, revealed notable influence by three variables among the placebo arms: cancer diagnosis, median age (Figure 1), and performance status. In contrast, we found no robust influence of study type (phase 1/2/3), measurable tumor, newly diagnosed versus relapsed/refractory, route of treatment, year of publication, previous lines of treatments, and total $\mathrm{n}$ of study population. SAEs were more common in studies with newly diagnosed tumors $(6.1 \%$ versus $4.9 \%)$, in studies that enrolled patients with measurable disease (26.3\%) versus no measurable disease $(9.8 \%)$, and in studies with male majority (22.7 vs. 10\%).

The middle age group of cohorts with a median age between 45 and 50 had the highest rate of headache reporting $(n=8$, mean $=20.6 \%, \mathrm{SD}=9.2$ ), whereas the next younger or older group 


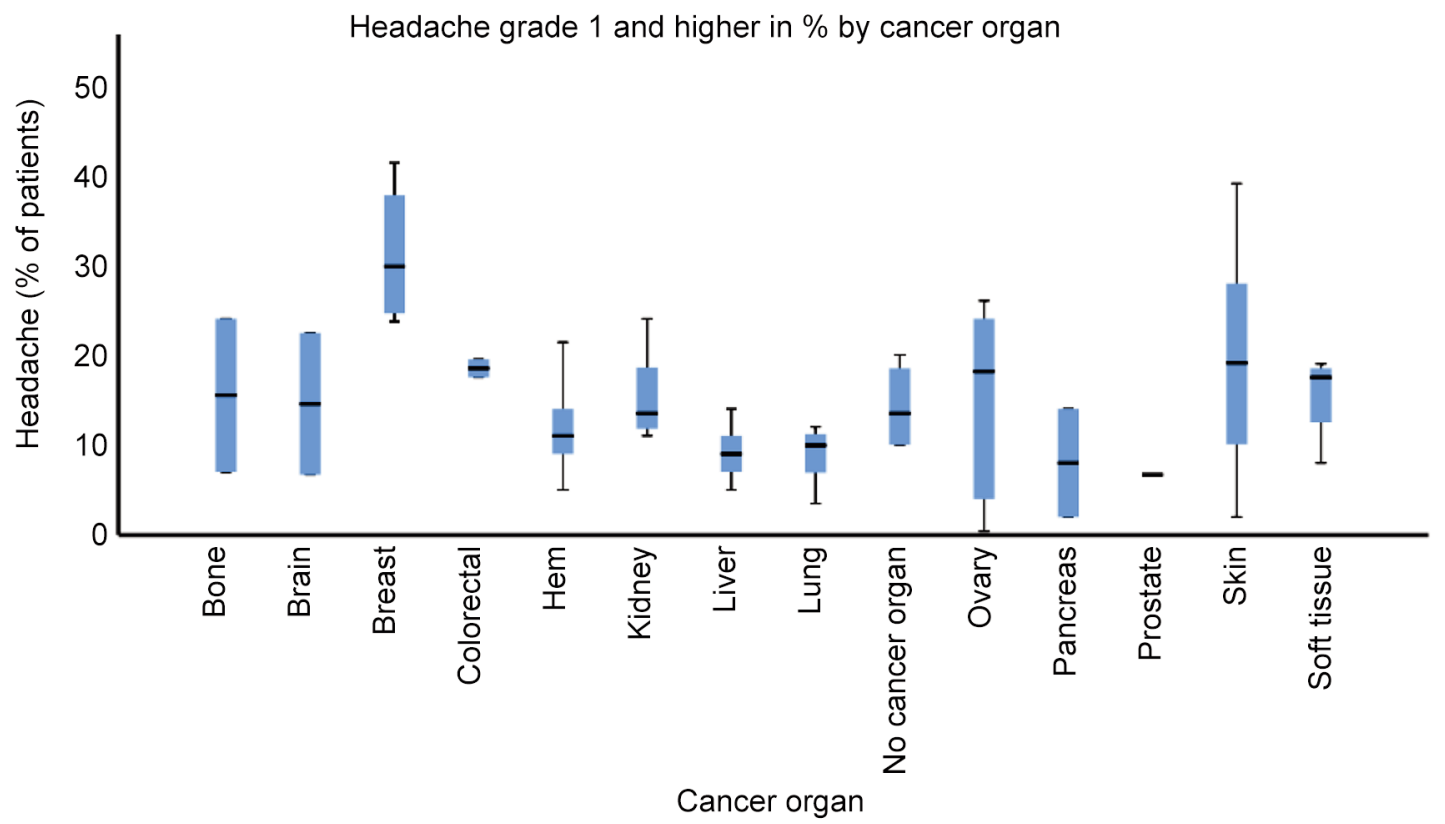

Figure 3. Cancer diagnosis and headache, in treatment and placebo arms.

had notable lower rates $(<45$ years of age: $n=6$, average $=14.5$, $\mathrm{SD}=7.3 ; 50-65$ years: $\mathrm{n}=18$, average $=9.8, \mathrm{SD}=5.5$ exploratory $p=0.004$ ANOVA). Repeating the analyses for headache frequency including all cohorts (treatment and placebo) resulted in the same trends, similar numbers, and even lower $p$-value (0.000009). In contrast, SAEs were most common in the oldest age group (placebo cohorts with median age $>65$ years: $18.3 \%$ ).

Performance status (PS) was commonly reported as ECOG PS (12). A healthy person is documented in these categories as "ECOG 0". In linear regression, the frequency of $\mathrm{ECOG}=0$ correlated with headache reporting (Pearson correlation $0.497, p=0.036, \mathrm{n}=18$, Figure 2A) indicating more headaches in persons with normal PS. There was no such correlation among the corresponding treatment cohorts; and the SAE frequency had the opposite correlation: more SAEs reported among cohorts with lower PS (Pearson correlation $-0.4, p=0.014$, Figure $2 \mathrm{~B}$ ).

The tumor diagnosis had a large influence on all of the AEs: Breast cancer had the highest rate of headache followed by colorectal carcinoma, and the lowest was pancreatic carcinoma (Figure 3). This distribution was similar in the placebo and non-placebo arms. Interestingly, the distribution of SAEs had a largely different pattern among diagnoses.

\section{Discussion}

We found that the rate of AEs reported in placebo-treated subjects was related to demographics. Cancer diagnosis, median age, and PS were particularly impactful for headache reporting. The frequency of ECOG 0 correlated with the frequency of headache reporting, indicating a higher frequency of headache among healthier subjects. The frequency of SAE was inversely correlated: higher among patients with poor PS.

The decision to discontinue treatment for AEs implies the assumption of causality. If the drug is placebo, then this assumption was an error. This phenomenon occurred in $5 \%$. In the treatment arms of the same studies, the rate was $15 \%$. In analogy, this suggests that among studies without randomization and control arm, an investigator's opinion of causality to the drug is correct only in two thirds of the cases.

We found patients with higher tumor burden, later treatment line, reduced PS, and higher age to be more likely to experience SAEs. This confirms the intuitive prediction that patients with more severe illness are also more likely to be admitted to the hospital, and validates the method. However, counterintuitively, headache was reported more often with a higher PS. This might be explained by reporting fatigue in the context of high symptom burden: Severe complications and symptoms of the underlying malignancy might make headache less relevant to patients and investigators. The same phenomenon might explain the lower headache reporting in elderly, and it might have an impact on the disease specific findings.

It is not surprising that the pattern of AEs depends on the underlying diagnosis: Myelodysplastic syndrome patients with thrombocytopenia report a high rate of skin hemorrhages (11). Others have shown high $\mathrm{I}^{2}$ values for placebo arms, indicating large heterogeneity and a general 
correlation of AEs between treatment and placebo control (5). Our data confirmed these findings; and they are very consistent with the well-known fact that symptoms are specific to the cancer organ. It is harder to explain why headache is more common among breast cancer patients, and why brain tumor patients rank among the lowest when it comes to headache reporting (Figure 3) (12). Exhaustion from reporting in clinical trials may potentially also explain this finding: Some patients might consider it irrelevant to report headaches to their physician, when she/he already knows that the patient has a brain tumor.

The data reported here support a novel approach of utilizing external controls to interpret $\mathrm{AE}$ data of single arm studies. However, given the large heterogeneity, a single bench mark AE frequency should be discouraged versus a model that takes demographics into account. For headache, such models should include tumor diagnosis, median age, and PS. An SAE model should also include: the line of treatment (newly diagnosed versus RR), and the frequency of measurable tumors.

\section{Conflicts of Interest}

The Authors have no conflicts of interest to declare regarding this study. JW is an employee of AbbVie; MW is an employee of Microsoft. However, this project was not part of the employments.

\section{Authors' Contributions}

BW: Idea, analysis, text, figures; MW: analysis, text; JW: idea, data collections, analysis, text, finishing communications.

\section{Acknowledgements}

The Authors would like to thank Patrick Boehnke, and Ruben Wolff for the helpful discussions.

\section{References}

1 Hinrichs MJM, Ryan PM, Zheng B, Afif-Rider S, Yu XQ, Gunsior M, Zhong H, Harper J, Bezabeh B, Vashisht K, Rebelatto M, Reed M, Ryan PC, Breen S, Patel N, Chen C, Masterson L, Tiberghien A, Howard PW, Dimasi N and Dixit R: Fractionated dosing improves preclinical therapeutic index of pyrrolobenzodiazepine-containing antibody drug conjugates. Clin Cancer Res 23(19): 5858-5868, 2017. PMID: 28630216. DOI: 10.1158/1078-0432.CCR-17-0219

2 Raju GK, Gurumurthi K, Domike R, Singh H, Weinstock C, Kluetz P, Pazdur R and Woodcock J: Using a benefit-risk analysis approach to capture regulatory decision making: renal cell carcinoma. Clin Pharmacol Ther 107(3): 495-506, 2020 PMID: 31355920. DOI: 10.1002/cpt.1589

3 Sisk BA, Dubois J, Hobbs BP and Kodish E: Reprioritizing risk and benefit: The future of study design in early-phase cancer research. Ethics Hum Res 41(6): 2-11, 2019. PMID: 31743629. DOI: 10.1002/eahr.500033
4 Ruiz-Schutz VC, Gomes LM, Mariano RC, de Almeida DVP, Pimenta JM, Dal Molin GZ, Kater FR, Yamamura R, Correa Neto NF, Maluf FC and Schutz FA: Risk of fatigue and anemia in patients with advanced cancer treated with olaparib: A metaanalysis of randomized controlled trials. Crit Rev Oncol Hematol 141: 163-173, 2019. PMID: 31306986. DOI: 10.1016/ j.critrevonc.2019.06.012

5 Chacón MR, Enrico DH, Burton J, Waisberg FD and Videla VM: Incidence of placebo adverse events in randomized clinical trials of targeted and immunotherapy cancer drugs in the adjuvant setting: a systematic review and meta-analysis. JAMA Netw Open 1(8): e185617, 2018. PMID: 30646278. DOI: 10.1001/ jamanetworkopen.2018.5617

6 Valle JW, Borbath I, Rosbrook B, Fernandez K and Raymond E: Sunitinib in patients with pancreatic neuroendocrine tumors: update of safety data. Future Oncol 15(11): 1219-1230, 2019. PMID: 30701988. DOI: 10.2217/fon-2018-0882

7 Neyeloff JL, Fuchs SC and Moreira LB: Meta-analyses and Forest plots using a microsoft excel spreadsheet: step-by-step guide focusing on descriptive data analysis. BMC Res Notes 5: 52, 2012. PMID: 22264277. DOI: 10.1186/1756-0500-5-52

8 Seitz L, Jin L, Leleti M, Ashok D, Jeffrey J, Rieger A, Tiessen RG, Arold G, Tan JBL, Powers JP, Walters MJ and Karakunnel J: Safety, tolerability, and pharmacology of AB928, a novel dual adenosine receptor antagonist, in a randomized, phase 1 study in healthy volunteers. Invest New Drugs 37(4): 711-721, 2019. PMID: 30569245. DOI: 10.1007/s10637-018-0706-6

9 Coleman R, Finkelstein DM, Barrios C, Martin M, Iwata H, Hegg R, Glaspy J, Periañez AM, Tonkin K, Deleu I, Sohn J, Crown J, Delaloge S, Dai T, Zhou Y, Jandial D and Chan A: Adjuvant denosumab in early breast cancer (D-CARE): an international, multicentre, randomised, controlled, phase 3 trial. Lancet Oncol 21(1): 60-72, 2020. PMID: 31806543. DOI: 10.1016/S1470-2045(19)30687-4

10 Oken MM, Creech RH, Tormey DC, Horton J, Davis TE, McFadden ET and Carbone PP: Toxicity and response criteria of the Eastern Cooperative Oncology Group. Am J Clin Oncol 5(6): 649-655, 1982. PMID: 7165009.

11 Mittelman M, Platzbecker U, Afanasyev B, Grosicki S, Wong RSM, Anagnostopoulos A, Brenner B, Denzlinger C, Rossi G, Nagler A, Garcia-Delgado R, Portella MSO, Zhu Z and Selleslag D: Eltrombopag for advanced myelodysplastic syndromes or acute myeloid leukaemia and severe thrombocytopenia (ASPIRE): a randomised, placebo-controlled, phase 2 trial. Lancet Haematol 5(1): e34-e43, 2018. PMID: 29241762. DOI: 10.1016/S2352-3026(17)30228-4

12 Wen PY, Reardon DA, Armstrong TS, Phuphanich S, Aiken RD, Landolfi JC, Curry WT, Zhu JJ, Glantz M, Peereboom DM, Markert JM, LaRocca R, O'Rourke DM, Fink K, Kim L, Gruber M, Lesser GJ, Pan E, Kesari S, Muzikansky A, Pinilla C, Santos RG and Yu JS: A randomized double-blind placebo-controlled phase ii trial of dendritic cell vaccine ICT-107 in newly diagnosed patients with glioblastoma. Clin Cancer Res 25(19): 5799-5807, 2019. PMID: 31320597. DOI: 10.1158/10780432.CCR-19-0261

Received March 20, 2021

Revised April 20, 2021 Accepted April 26, 2021 